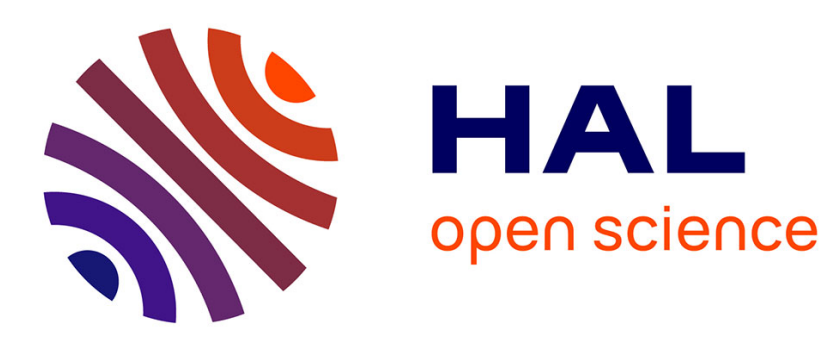

\title{
Higher-order averaging for DC-DC converters
}

Thomas Devos, Philippe Martin

\section{To cite this version:}

Thomas Devos, Philippe Martin. Higher-order averaging for DC-DC converters. IECON 2019 45th Annual Conference of the IEEE Industrial Electronics Society, Oct 2019, Lisbon, Portugal. 10.1109/IECON.2019.8927089 . hal-02413777

\section{HAL Id: hal-02413777 https://hal.science/hal-02413777}

Submitted on 19 Dec 2019

HAL is a multi-disciplinary open access archive for the deposit and dissemination of scientific research documents, whether they are published or not. The documents may come from teaching and research institutions in France or abroad, or from public or private research centers.
L'archive ouverte pluridisciplinaire HAL, est destinée au dépôt et à la diffusion de documents scientifiques de niveau recherche, publiés ou non, émanant des établissements d'enseignement et de recherche français ou étrangers, des laboratoires publics ou privés. 


\section{Higher-order averaging for DC-DC converters}

\author{
Thomas Devos \\ Industrial Automation Business \\ Schneider Electric \\ Pacy-sur-Eure, France \\ thomas.devos@se.com
}

\author{
Philippe Martin \\ Centre Automatique et Systèmes, MINES ParisTech \\ PSL Research University \\ Paris, France \\ philippe.martin@mines-paristech.fr
}

\begin{abstract}
We revisit higher-order averaging and ripple computation for DC-DC converters in Continuous Conduction Mode, following the modern treatment of periodic averaging theory. We give a much simpler treatment of second-order averaging and ripple computation than usually found in the power electronics literature. We also extend it to third-order averaging, thus significantly improving the accuracy of the approximations, which is useful in particular when the switching frequency is not very high.
\end{abstract}

Index Terms-DC-DC converters, ripple analysis, higher-order averaging.

\section{INTRODUCTION}

A switched electronic system such as a DC-DC converter is difficult to analyze and to some extent to simulate. This is due to the fact that the underlying mathematical model is a "composite" differential system, with one subsystem for each configuration of the switches. A now standard analysis tool is "plain" (i.e., first-order) averaging, which consists in approximating this composite system by a single one describing the "averaged" behavior, see e.g. [1, section 16.2]. Unfortunately, first-order averaging is often not accurate enough, because it doesn't capture the ripple, which may be fairly important unless the switching frequency is very high. In this case, it is nevertheless possible to obtain a better approximation by computing the ripple thanks to second-order averaging, see in particular [2]-[8]. The approach, based on the so-called Krylov-Bogoliubov-Mitropolsky method of averaging [9], is considered quite complicated, hence less widely used as plain averaging.

In this paper, we revisit higher-order averaging and ripple computation for DC-DC converters in Continuous Conduction Mode (CCM), following a more modern treatment of periodic averaging theory as presented e.g. in [10, section 2.9]. The contribution with respect to the power electronics literature is mainly two-fold:

- a much simpler approach to second-order averaging and computation of the corresponding ripple

- the extension to third-order averaging along the same lines, with more complicated but still reasonably simple calculations. This yields better approximations than the second-order case, which can be useful for instance when a great accuracy is needed or when the switching frequency is low.
As a by-product, the asymptotic accuracy of the approximation obtained by averaging is clearly stated, which is usually not done in the power electronics literature.

The paper runs as follows: section II presents the general model of a DC-DC converter which is considered, together with some preliminary material; section III summarizes the modern theory of higher-order periodic averaging, and applies it to the DC-DC converter; finally, section IV illustrates the interest of the approach with numerical simulations of an ideal boost converter.

We list below some definitions and basic facts used throughout the paper:

- if $f$ is a $T$-periodic function, its mean is the constant $\bar{f}$ defined by $\bar{f}:=\frac{1}{T} \int_{0}^{T} f(\sigma) d \sigma$

- if $f$ is a $T$-periodic function with zero mean, any primitive of $f$ is also $T$-periodic (this is not true if $\bar{f} \neq 0$ )

- if $f$ is a $T$-periodic function with zero mean, its (unique) primitive with zero mean is the $T$-periodic function $f_{1}$ defined by

$$
f_{1}(\tau)=\int_{0}^{\tau} f(\sigma) d \sigma-\frac{1}{T} \int_{0}^{T} \int_{0}^{\tau} f(\sigma) d \sigma d \tau
$$

- $\mathcal{O}$ is the "Big-O" notation of analysis: if $\alpha$ and $\beta$ are two functions, $\alpha(\varepsilon)=\mathcal{O}(\beta(\varepsilon))$ means $|\alpha(\varepsilon)| \leq C|\beta(\varepsilon)|$ for some positive constant $C$ when $\varepsilon \rightarrow 0$.

\section{General MOdel of A DC-DC CONVERTER}

\section{A. Putting a DC-DC converter into standard form}

A DC-DC converter in CCM can generally be modeled by a differential system of the form

$$
\frac{d x}{d t}=\left(A_{1} x+a_{1}\right) q(t)+\left(A_{2} x+a_{2}\right)(1-q(t)),
$$

where the so-called switching function $q$ is $T$-periodic; $A_{1}$ and $A_{2}$ are constant $n \times n$ matrices, $a_{1}$ and $a_{2}$ are constant $n \times 1$ vectors. It will be convenient for averaging to first put (1) into the normalized form

$$
\frac{d X}{d \tau}=\varepsilon\left((A X+a)+(B X+b) s_{0}(\tau)\right),
$$

where $\varepsilon$ is a small parameter and $s_{0}$ is $2 \pi$-periodic with zero mean. To this end, we first rewrite (1) as

$$
\begin{aligned}
\frac{d x}{d t}= & \left(\bar{q} A_{1}+(1-\bar{q}) A_{2}\right) x+\bar{q} a_{1}+(1-\bar{q}) a_{2} \\
& +\left(\left(A_{1}-A_{2}\right) x+a_{1}-a_{2}\right)(q(t)-\bar{q}),
\end{aligned}
$$


where the first line of the rhs is independent of time and the second line depends on time only through the zero mean function $q(t)-\bar{q}$. We next perform the change of variables $X(\tau)=P x(t)$, where $P$ is a suitable invertible matrix (in general diagonal), and the time change $\tau:=\frac{2 \pi t}{T}$. This yields the desired form (3), with

$$
\begin{aligned}
\varepsilon & :=\frac{T}{2 \pi \lambda} \\
s_{0}(\tau) & :=q\left(\frac{T}{2 \pi} \tau\right) \\
A & :=\lambda P\left(\bar{q} A_{1}+(1-\bar{q}) A_{2}\right) P^{-1} \\
a & :=\lambda P\left(\bar{q} a_{1}+(1-\bar{q}) a_{2}\right) \\
B & :=\lambda P\left(A_{1}-A_{2}\right) P^{-1} \\
b & :=\lambda P\left(a_{1}-a_{2}\right) ;
\end{aligned}
$$

$\lambda$ is a scaling factor, chosen so as to balance the entries of $A$.

Remark 1: In the power electronics literature on averaging, it is common to consider instead of (1) the composite system

$$
\begin{aligned}
& \frac{d x}{d t}=A_{1} x+a_{1} \quad \text { when the switch is on (i.e., } q(t)=1 \text { ) } \\
& \frac{d x}{d t}=A_{2} x+a_{2} \quad \text { when the switch is off (i.e., } q(t)=0 \text { ). }
\end{aligned}
$$

Working with a single differential system containing a switching function such as (1) or (2) makes (higher-order) averaging easier to handle, because it fits directly into the mathematical framework of the theory.

Remark 2: The normalized form (2) is by no mean unique, nor even a mandatory step for performing averaging in practice. It is rather a normalization step that highlights a small parameter $\varepsilon$ which motivates the averaging analysis. In theory, the averaging process must be conducted on the normalized form to be justified, and only then can everything be expressed back into the original variables; in practice, it could be conducted directly on the original system (1), provided one is careful in evaluating what is "small" and what isn't (which is not so easy when considering non-normalized quantities).

\section{B. Example: ideal boost converter}

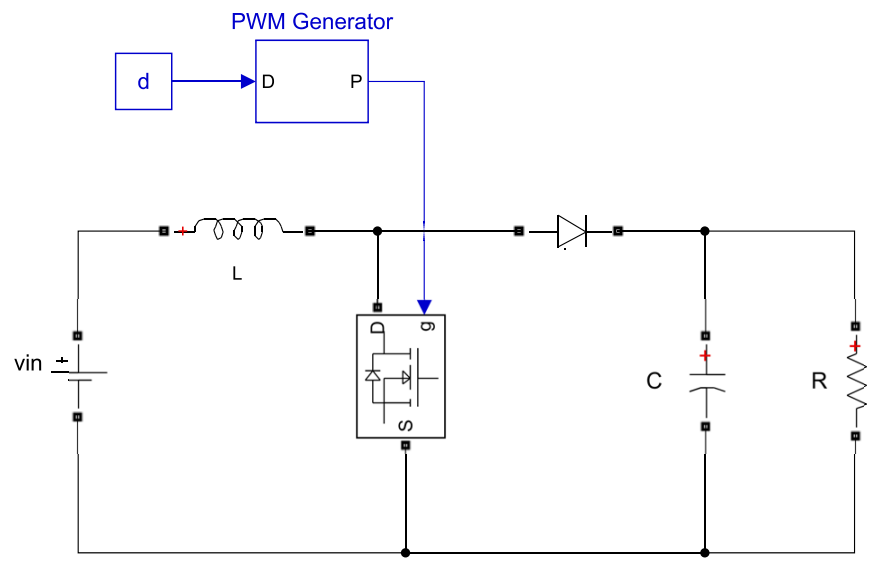

Figure 1. Ideal boost converter.
We illustrate the normalization step of section II-A on the ideal boost converter of Fig. 1. The converter is modeled by

$$
\begin{aligned}
L \frac{d \imath}{d t} & =v_{i n} q(t)+\left(v_{i n}-v\right)(1-q(t)) \\
C \frac{d v}{d t} & =\imath(1-q(t))-\frac{v}{R},
\end{aligned}
$$

where $\imath$ is the current into the inductor $L$ and $v$ is the voltage across the capacitor $C$; the switching function applied to the transistor is the standard PWM signal with (constant) duty ratio $d$ and frequency $\frac{1}{T}$

$$
q(t)= \begin{cases}1, & 0<t \quad \bmod T \leq T d \\ 0, & T d<t \quad \bmod T \leq T\end{cases}
$$

and satisfies $\bar{q}=d$.

Changing then time and variables according to

$$
\begin{aligned}
\tau & :=2 \pi \frac{t}{T} \\
I & :=\sqrt{\frac{\frac{L}{R}}{R C}} \frac{R \imath}{v_{i n}}=\sqrt{\frac{L}{C}} \frac{\imath}{v_{i n}} \\
V & :=\frac{v}{v_{i n}}
\end{aligned}
$$

and selecting the scaling factor $\lambda:=\frac{\sqrt{L C}}{1-d}$, we readily find

$$
\begin{aligned}
\frac{d I}{d \tau} & =\varepsilon\left(-V+\frac{1}{1-d}+V s_{0}(\tau)\right) \\
\frac{d V}{d \tau} & =\varepsilon\left(I-\mu V-I s_{0}(\tau)\right)
\end{aligned}
$$

with

$$
\begin{aligned}
\varepsilon & :=\frac{T(1-d)}{2 \pi \sqrt{L C}} \\
\mu & :=\frac{1}{R(1-d)} \sqrt{\frac{L}{C}} \\
s_{0}(\tau) & :=\frac{q(t)-d}{1-d} .
\end{aligned}
$$

The transformed system has the desired form (2), with $A=$

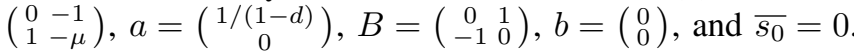

\section{Periodic functions shaping the ripple}

We will see in sections III-B and III-C that the state ripple is shaped by some periodic functions derived from $s_{0}$, namely

- $s_{1}$ (zero-mean primitive of $s_{0}$ ) for second-order averaging

- $s_{1}, s_{2}$ (zero mean primitive of $s_{1}$ ), and $\left(s_{0} s_{1}\right)_{1}$ (zero mean primitive of $\left.s_{0} s_{1}\right)$ for third-order averaging.

As an example, we compute here these functions in the case of the standard PWM switching function $q$ of section II-B. They are displayed in Fig. 2 for the case $d:=0.7$.

From the definition of the switching function $q$, we have

$$
s_{0}(\tau)= \begin{cases}1, & 0<\frac{\tau}{2 \pi} \leq d \\ \frac{-d}{1-d}, & d<\frac{\tau}{2 \pi} \leq 1\end{cases}
$$




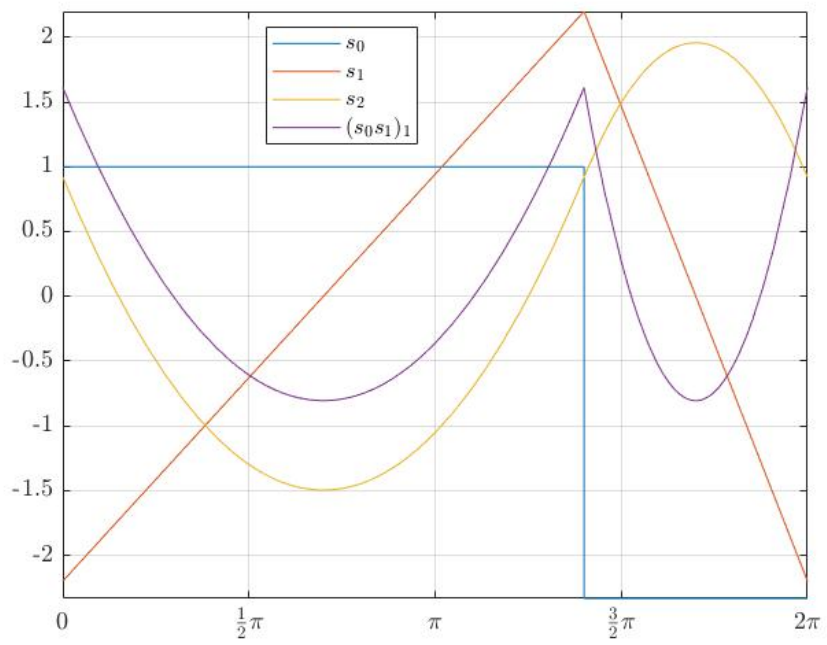

Figure 2. Periodic functions $s_{0}, s_{1}, s_{2}$, and $\left(s_{0} s_{1}\right)_{1}$ for $d:=0.7$.

where $\tau:=\tau \bmod 2 \pi$; clearly, $\overline{s_{0}}=0$. As it is somewhat awkward to manipulate a piecewise-defined function, we equivalently rewrite $s_{0}$ as

$$
s_{0}(\tau)=\frac{1-2 d-\operatorname{sign}(\boldsymbol{\tau}-2 \pi d)}{2(1-d)} .
$$

We then find

$$
\begin{aligned}
s_{1}(\tau)= & \int_{0}^{\tau} s_{0}(\sigma) d \sigma-\frac{1}{2 \pi} \int_{0}^{2 \pi} \int_{0}^{\tau} s_{0}(\sigma) d \sigma d \tau \\
= & \frac{(1-2 d) \tau-|\boldsymbol{\tau}-2 \pi d|+2 \pi d^{2}}{2(1-d)} \\
s_{2}(\tau)= & \int_{0}^{\tau} s_{1}(\sigma) d \sigma-\frac{1}{2 \pi} \int_{0}^{2 \pi} \int_{0}^{\tau} s_{1}(\sigma) d \sigma d \tau \\
= & \frac{(1-2 d) \tau^{2}-(\boldsymbol{\tau}-2 \pi d)|\boldsymbol{\tau}-2 \pi d|}{4(1-d)} \\
& +\frac{3 \pi d^{2} \boldsymbol{\tau}-\pi^{2} d\left(2 d^{2}+1\right)}{3(1-d)} \\
\left(s_{0} s_{1}\right)_{1}(\tau)= & \int_{0}^{\tau}\left(s_{0} s_{1}\right)(\sigma) d \sigma-\frac{1}{2 \pi} \int_{0}^{2 \pi} \int_{0}^{\tau}\left(s_{0} s_{1}\right)(\sigma) d \sigma d \tau \\
= & \frac{s_{1}^{2}(\tau)-\overline{s_{1}^{2}}}{2} \\
= & \frac{\left((1-2 d) \boldsymbol{\tau}-|\boldsymbol{\tau}-2 \pi d|+2 \pi d^{2}\right)^{2}}{8(1-d)^{2}}-\frac{\pi^{2} d^{2}}{6},
\end{aligned}
$$

where the last but one line stems from integrating by parts. It is easily seen that, as desired, $s_{1}^{\prime}=s_{0}, s_{2}^{\prime}=s_{1},\left(s_{0} s_{1}\right)_{1}^{\prime}=s_{0} s_{1}$, and $\overline{s_{1}}=\overline{s_{2}}=\overline{\left(s_{0} s_{1}\right)_{1}}=0$.

\section{HIGHER-ORDER AVERAGING}

We first summarize the theory of higher-order periodic averaging along the lines of [10, section 2.9]. We then apply it to the DC-DC converter; the second-order case turns out to be very simple, whereas the third-order case is more complicated but still easily tractable.

\section{A. A summary of higher-order periodic averaging theory}

Consider the differential system

$$
\frac{d X}{d \tau}=\varepsilon f(X, \tau)
$$

where $\varepsilon$ is a "small" parameter, and $f$ is $2 \pi$-periodic with respect to its second argument. The theory of $k^{t h}$-order averaging asserts it is possible to find a time-invariant system

$$
\frac{d \bar{X}}{d \tau}=\varepsilon g_{1}(\bar{X})+\cdots+\varepsilon^{k} g_{k}(\bar{X}),
$$

such that the solution $X(\tau)$ of (3) and the solution $\bar{X}(\tau)$ of (4) are related by

$$
X(\tau)=\bar{X}(\tau)+\sum_{i=1}^{k-1} \varepsilon^{i} u_{i}(\bar{X}(\tau), \tau)+\mathcal{O}\left(\varepsilon^{k}\right), \quad \tau>\tau_{0},
$$

for some suitable $u_{i} 2 \pi$-periodic with respect to their second arguments, provided their initial conditions are also related by

$$
X\left(\tau_{0}\right)=\bar{X}\left(\tau_{0}\right)+\sum_{i=1}^{k-1} \varepsilon^{i} u_{i}\left(\bar{X}\left(\tau_{0}\right), \tau_{0}\right)+\mathcal{O}\left(\varepsilon^{k}\right) .
$$

The $g_{i}$ 's and $u_{i}$ 's are not uniquely defined (there are infinitely many possible solutions), but for $g_{1}$, which is necessarily the time average of $f$, i.e., $g_{1}(\bar{X})=\frac{1}{2 \pi} \int_{0}^{2 \pi} f(\bar{X}, \tau) d \tau$. In the sequel, we will further impose the $u_{i}$ 's to have zero mean, which then uniquely defines them as well as the $g_{i}$ 's; in particular, this yields $g_{2}=0$. The physical reason for this choice is that the $\varepsilon^{i} u_{i}$ 's appear as small zero-mean "ripples" on top of the "averaged value" $\bar{X}$.

The proof comprises two main steps. The first step is to determine a so-called quasi-identity transformation

$$
X=\widetilde{X}+\sum_{i=1}^{k-1} \varepsilon^{i} u_{i}(\widetilde{X}, \tau)
$$

which puts the system into

$$
\frac{d \widetilde{X}}{d \tau}=\varepsilon g_{1}(\widetilde{X})+\cdots+\varepsilon^{k} g_{k}(\widetilde{X})+\varepsilon^{k} h(\widetilde{X}, \tau)+\mathcal{O}\left(\varepsilon^{k+1}\right)
$$

the whole point is that the transformed system does not depend on $\tau$ except through the zero-mean function $h$. This step is purely computational, and several solutions are possible. The second step is a comparison result. It asserts the averaged system (4) obtained by truncating (5) has in fact the same solution as (5) to order $\varepsilon^{k}$, starting from the same initial conditions; the remarkable point is that the term $\varepsilon^{k} h$ in (5) has therefore no influence at order $\varepsilon^{k}$ because it has zero mean. The overall conclusion then immediately follows from those two steps.

\section{B. Second-order averaging for DC-DC converters}

We now apply the general theory of section III-A to secondorder averaging of a DC-DC converter. We thus start with a system in the form (2), with initial condition $X\left(\tau_{0}\right):=X_{0}$. On the other hand, consider the time-invariant system

$$
\frac{d \bar{X}}{d \tau}=\varepsilon(A \bar{X}+a),
$$


with initial condition $\bar{X}\left(\tau_{0}\right):=\bar{X}_{0}$ given by

$$
X_{0}=\bar{X}_{0}+\varepsilon\left(B \bar{X}_{0}+b\right) s_{1}\left(\tau_{0}\right),
$$

where $s_{1}$ is the primitive of $s_{0}$ with zero-mean.

We will establish that the solution $X(\tau)$ of (2) and the solution $\bar{X}(\tau)$ of (6) are related by

$$
X(\tau)=\bar{X}(\tau)+\varepsilon(B \bar{X}(\tau)+b) s_{1}(\tau)+\mathcal{O}\left(\varepsilon^{2}\right) .
$$

$X(\tau)$ therefore decomposes as a "slowly-varying" part $\bar{X}(\tau)$ and a small periodic ripple $\varepsilon(B \bar{X}(\tau)+b) s_{1}(\tau)$ with "slowlyvarying" amplitude.

We first show the quasi-identity transformation

$$
X=\tilde{X}+\varepsilon(B \tilde{X}+b) s_{1}(\tau)
$$

puts (2) into

$$
\frac{d \widetilde{X}}{d \tau}=\varepsilon(A \widetilde{X}+a)+\varepsilon^{2} Z(\widetilde{X}, \tau)+\mathcal{O}\left(\varepsilon^{3}\right),
$$

where $Z$ is a term with zero mean yet to define. On the one hand, plugging (8) into the rhs of (2) yields

$$
\frac{d X}{d \tau}=\varepsilon\left((A \tilde{X}+a)+(B \widetilde{X}+b) s_{0}(\tau)\right)+\varepsilon^{2} Z_{1}(\tilde{X}, \tau),
$$

where

$$
Z_{1}(\widetilde{X}, \tau):=A(B \widetilde{X}+b) s_{1}(\tau)+B(B \widetilde{X}+b)\left(s_{0} s_{1}\right)(\tau)
$$

has zero mean. Indeed, $s_{1}$ has by construction zero mean, and

$$
\overline{s_{0} s_{1}}=\frac{1}{2 \pi}\left[\frac{s_{1}^{2}(\tau)}{2}\right]_{0}^{2 \pi}=0 .
$$

On the other hand, differentiating (8) yields

$$
\begin{aligned}
\frac{d X}{d \tau}= & \varepsilon\left((A \widetilde{X}+a)+(B \widetilde{X}+b) s_{0}(\tau)\right) \\
& +\varepsilon^{2} Z_{2}(\widetilde{X}, \tau)+\varepsilon^{2} Z(\widetilde{X}, \tau)+\mathcal{O}\left(\varepsilon^{3}\right),
\end{aligned}
$$

where $Z_{2}(\tilde{X}, \tau):=B(A \widetilde{X}+a) s_{1}(\tau)$ has obviously zero mean. Setting then $Z:=Z_{1}-Z_{2}$, we find that (10) and (11) agree to order $\varepsilon^{2}$. That is, (8) transforms (2) into (9), with $Z$ by construction zero mean.

We conclude by using the comparison result: since $Z$ has zero mean, the averaged system (6) has the same solution as (9) to order $\varepsilon^{2}$, which directly implies the claim.

Remark 3: As mentioned in section III-B, the averaged system (6) has no term in $\varepsilon^{2}$ because the transformation (hence the ripple) has zero mean.

\section{Third-order averaging for DC-DC converters}

Similarly, but with more tedious calculations, we can handle third-order averaging for a DC-DC converter. We start as before with a system in the form (2), with initial condition $X\left(\tau_{0}\right):=X_{0}$. On the other hand, we consider the timeinvariant system

$\frac{d \bar{X}}{d \tau}=\varepsilon(A \bar{X}+a)-\varepsilon^{3} B((A B-B A) \bar{X}+A b-B a) \overline{s_{1}^{2}}$, with initial condition $\bar{X}\left(\tau_{0}\right):=\bar{X}_{0}$ given by

$$
\begin{aligned}
X_{0}= & \bar{X}_{0}+\varepsilon\left(B \bar{X}_{0}+b\right) s_{1}\left(\tau_{0}\right) \\
& +\varepsilon^{2}\left((A B-B A) \bar{X}_{0}+A b-B a\right) s_{2}\left(\tau_{0}\right) \\
& +\varepsilon^{2} B\left(B \bar{X}_{0}+b\right)\left(s_{0} s_{1}\right)_{1}\left(\tau_{0}\right)
\end{aligned}
$$

$s_{2}$ is the primitive of $s_{1}$ with zero-mean, and $\left(s_{0} s_{1}\right)_{1}$ the primitive of $s_{0} s_{1}$ with zero-mean.

We will establish that the solution $X(\tau)$ of (2) and the solution $\bar{X}(\tau)$ of (12) are related by

$$
\begin{aligned}
X(\tau)= & \bar{X}(\tau)+\varepsilon(B \bar{X}(\tau)+b) s_{1}(\tau) \\
& +\varepsilon^{2}((A B-B A) \bar{X}(\tau)+A b-B a) s_{2}(\tau) \\
& +\varepsilon^{2} B(B \bar{X}+b)\left(s_{0} s_{1}\right)_{1}(\tau)+\mathcal{O}\left(\varepsilon^{3}\right)
\end{aligned}
$$

Thus, on top of the first-order ripple $\varepsilon(B \bar{X}(\tau)+b) s_{1}(\tau)$, there is also a second-order ripple modulated by $s_{2}$ and $\left(s_{0} s_{1}\right)_{1}$. Notice also that the averaged system (12) has a term in $\varepsilon^{3}$ but as usual no term in $\varepsilon^{2}$.

We first show the quasi-identity transformation

$$
\begin{aligned}
X(\tau)= & \tilde{X}(\tau)+\varepsilon(B \tilde{X}(\tau)+b) s_{1}(\tau) \\
& +\varepsilon^{2}((A B-B A) \widetilde{X}(\tau)+A b-B a) s_{2}(\tau) \\
& +\varepsilon^{2} B(B \widetilde{X}+b)\left(s_{0} s_{1}\right)_{1}(\tau)
\end{aligned}
$$

puts (2) into

$$
\begin{aligned}
\frac{d \tilde{X}}{d \tau}= & \varepsilon(A \widetilde{X}+a)-\varepsilon^{3} B((A B-B A) \widetilde{X}+A b-B a) \overline{s_{1}^{2}} \\
& +\varepsilon^{3} Z(\widetilde{X}, \tau)+\mathcal{O}\left(\varepsilon^{4}\right)
\end{aligned}
$$

where $Z$ is a term with zero mean yet to define. On the one hand, plugging (14) into the rhs of (2) yields

$$
\begin{aligned}
\frac{d X}{d \tau}= & \varepsilon\left((A \widetilde{X}+a)+(B \widetilde{X}+b) s_{0}(\tau)\right) \\
& +\varepsilon^{2}\left(A+B s_{0}(\tau)\right)(B \widetilde{X}+b) s_{1}(\tau) \\
& +\varepsilon^{3} B((A B-B A) \widetilde{X}+A b-B a) \overline{s_{0} s_{2}} \\
& +\varepsilon^{3} Z_{1}(\widetilde{X}, \tau),
\end{aligned}
$$

where

$$
\begin{aligned}
Z_{1}(\widetilde{X}, \tau):= & A((A B-B A) \widetilde{X}+A b-B a) s_{2}(\tau) \\
& +A B(B \widetilde{X}+b)\left(s_{0} s_{1}\right)_{1}(\tau) \\
& +B((A B-B A) \widetilde{X}+A b-B a)\left(\left(s_{0} s_{2}\right)(\tau)-\overline{s_{0} s_{2}}\right) \\
& +B^{2}(B \widetilde{X}+b)\left(s_{0}\left(s_{0} s_{1}\right)_{1}\right)(\tau)
\end{aligned}
$$

has zero mean. Indeed, $s_{2},\left(s_{0} s_{1}\right)_{1}$ and $s_{0} s_{2}-\overline{s_{0} s_{2}}$ have by construction zero mean; moreover, integrating by parts gives

$$
\begin{aligned}
\overline{s_{0}\left(s_{0} s_{1}\right)_{1}} & =\frac{1}{2 \pi}\left[\left(s_{1}\left(s_{0} s_{1}\right)_{1}\right)(\tau)\right]_{0}^{2 \pi}-\overline{s_{1}\left(s_{0} s_{1}\right)} \\
& =0-\frac{1}{2 \pi}\left[\frac{s_{1}^{3}(\tau)}{3}\right]_{0}^{2 \pi} \\
& =0 .
\end{aligned}
$$



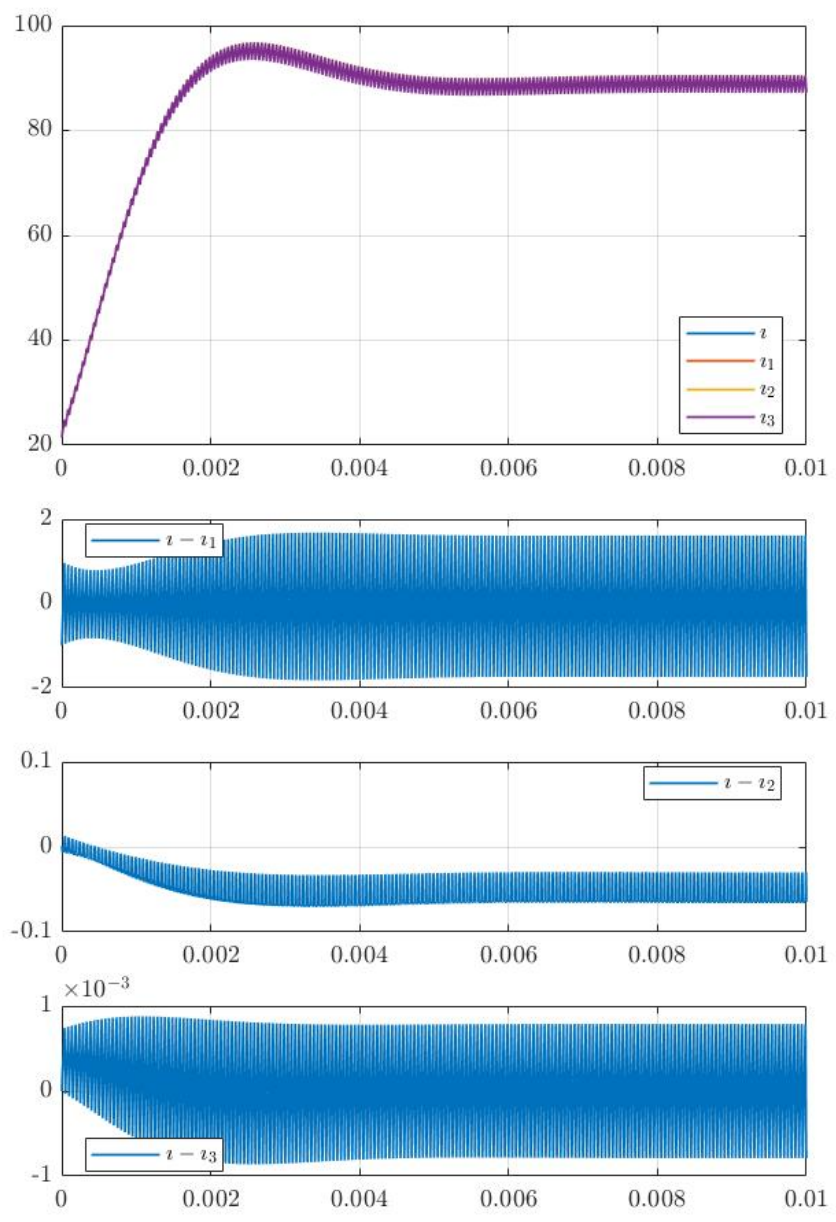

Figure 3. Currents $\imath, \imath_{1}, \imath_{2}, \imath_{3}$ at $20 \mathrm{kHz}$.

On the other hand, differentiating (14) yields

$$
\begin{aligned}
\frac{d X}{d \tau}= & \varepsilon\left((A \tilde{X}+a)+(B \tilde{X}+b) s_{0}(\tau)\right) \\
& +\varepsilon^{2}\left(A+B s_{0}(\tau)\right)(B \widetilde{X}+b) s_{1}(\tau) \\
& -\varepsilon^{3} B((A B-B A) \widetilde{X}+A b-B a) \overline{s_{1}^{2}} \\
& +\varepsilon^{3} Z_{2}(\widetilde{X}, \tau)+\varepsilon^{3} Z(\widetilde{X}, \tau)+\mathcal{O}\left(\varepsilon^{4}\right),
\end{aligned}
$$

where

$$
\begin{aligned}
Z_{2}(\widetilde{X}, \tau):= & B^{2}(A \widetilde{X}+a)\left(s_{0} s_{1}\right)_{1}(\tau) \\
& +(A B-B A)(A \widetilde{X}+a) s_{2}(\tau)
\end{aligned}
$$

has obviously zero mean. Setting then $Z:=Z_{1}-Z_{2}$, we find that (16) and (17) agree to order $\varepsilon^{3}$. That is, (14) transforms (2) into (15), with $Z$ by construction zero mean.

We conclude once again by using the comparison result: since $Z$ has zero mean, the averaged system (12) has the same solution as (15) to order $\varepsilon^{3}$, which directly implies the claim.

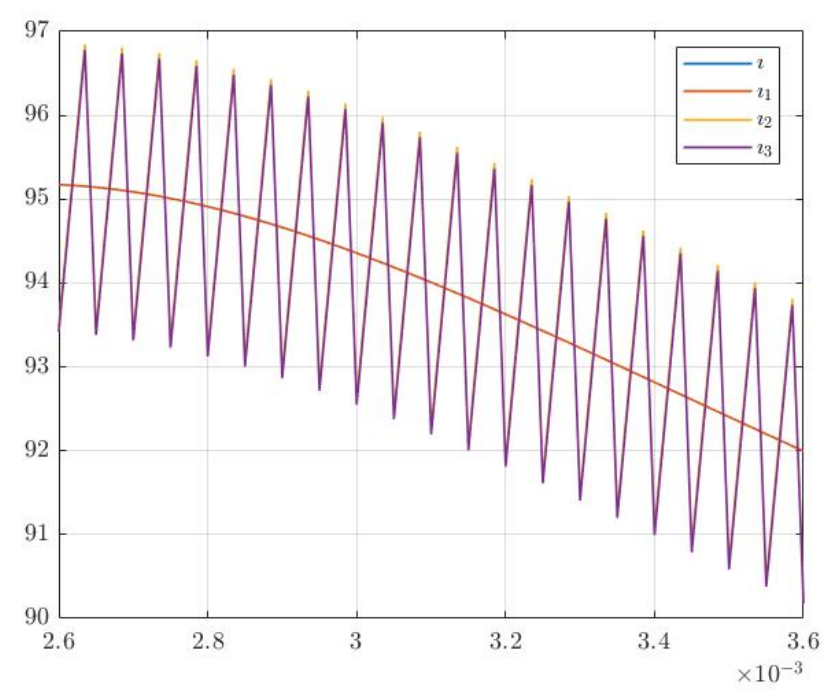

Figure 4. Currents $\imath, \imath_{1}, \imath_{2}, \imath_{3}$ at $20 \mathrm{kHz}$ (zoom).

\section{NUMERICAL SIMULATIONS: IDEAL BOOST CONVERTER}

We illustrate the results of the previous sections on the ideal boost converter of section II-B, with values $R=3 \Omega, L=$ $250 \mu \mathrm{H}, C=200 \mu \mathrm{F}, d=0.7$, and $v_{i n}=24 \mathrm{~V}$. The system was first normalized as explained in section II-B, then the second- and third-order averaging procedures were applied; finally the resulting equations were expressed back into the original variables, and simulated with Matlab-Simulink. The averaged system for second-order averaging is given by (6) and the ripple by (7); the averaged system for third-order averaging is given by (12) and the ripple by (13). The periodic functions appearing in these expressions are computed in section II-C.

The first numerical experiment was performed with the PWM frequency $\frac{1}{T}=20 \mathrm{kHz}$. Fig. 3 displays the evolution of the currents: $\imath$ is the true value of the current, $\imath_{1}$ is its approximation with plain first-order averaging (i.e., the solution of (6) with no ripple correction), $\imath_{2}$ its approximation with second-order averaging, and $\imath_{3}$ its approximation with third-order averaging. As expected, the errors between the true and approximated currents significantly decrease with the averaging order. Fig. 4 provides a closer view around $t=3 \mathrm{~ms}$; the ripple is very close to the triangular shape of $s_{1}$, because the $\varepsilon^{2}$-ripple is very small. Fig. 5 and Fig. 6 show the corresponding information for the voltages.

To better highlight the interest of third-order averaging, the same numerical experiment was performed with the rather low PWM frequency $\frac{1}{T}=2 \mathrm{kHz}$ : the approximations given by second-order averaging are now noticeably off the true values, whereas the approximations given by third-order averaging are still very good, see Fig. 7 and 9; besides the ripple is now only vaguely triangular, because the $\varepsilon^{2}$-ripple is no longer negligible, see Fig. 8 and 10.

Notice the errors between the true and approximated values behave quantitatively according to the theory: $10^{1}$ times smaller at $20 \mathrm{kHz}$ than at $2 \mathrm{kHz}$ for first-order averaging, 

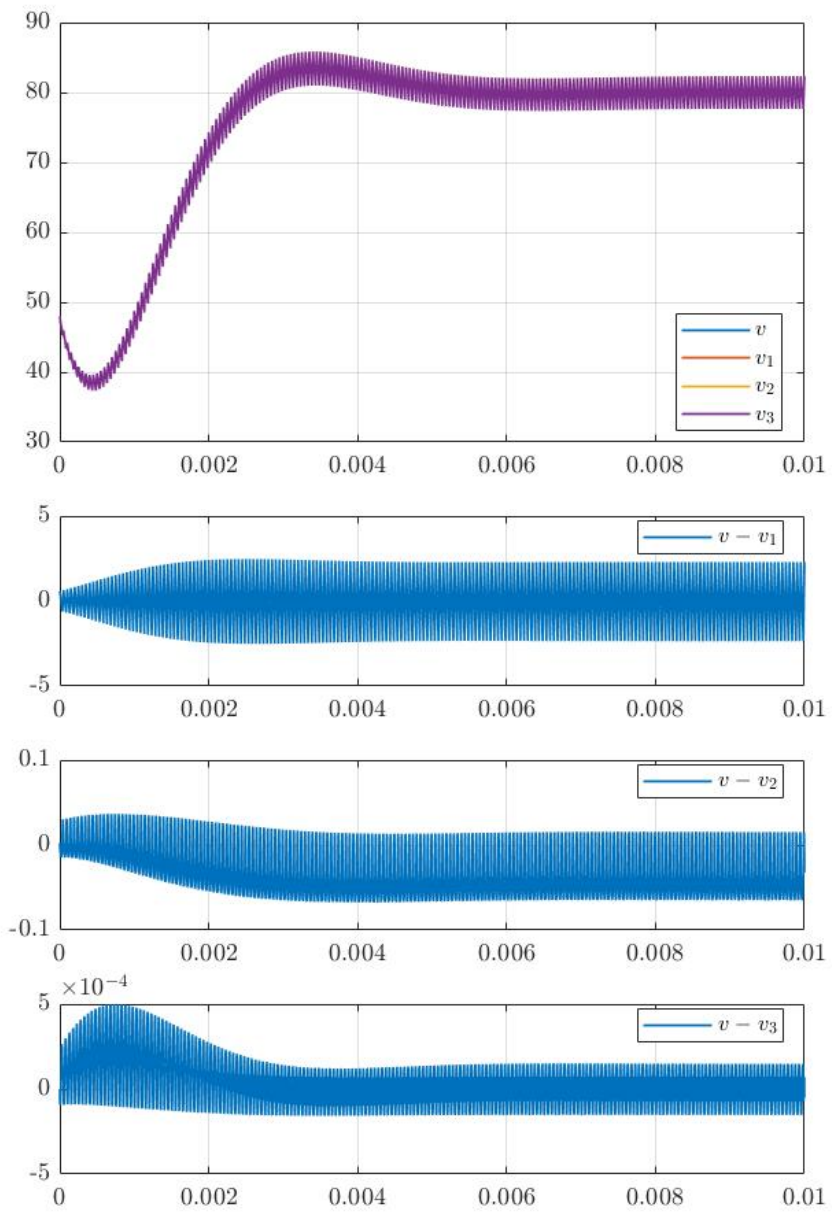

Figure 5. Voltages $v, v_{1}, v_{2}, v_{3}$ at $20 \mathrm{kHz}$.

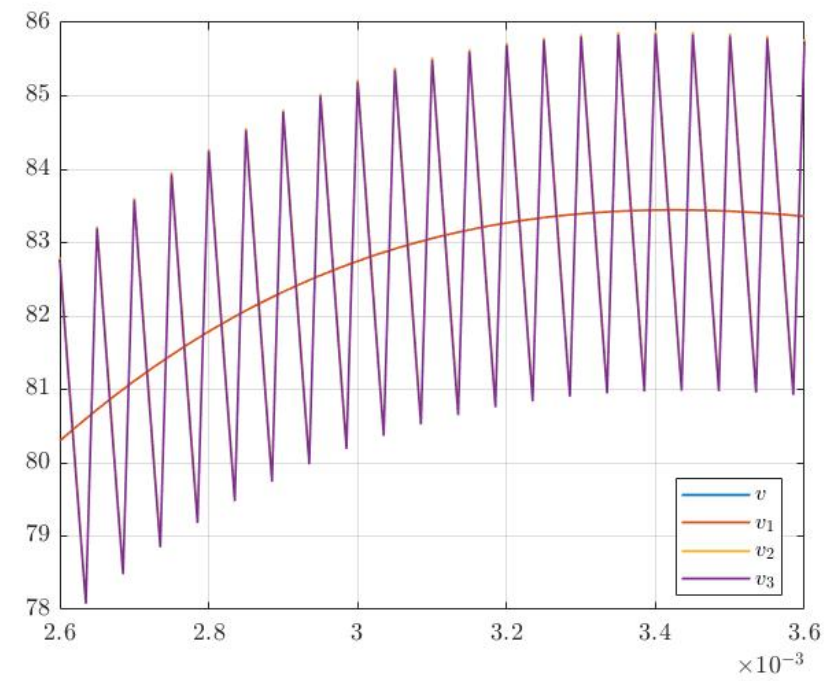

Figure 6. Voltages $v, v_{1}, v_{2}, v_{3}$ at $20 \mathrm{kHz}$ (zoom).
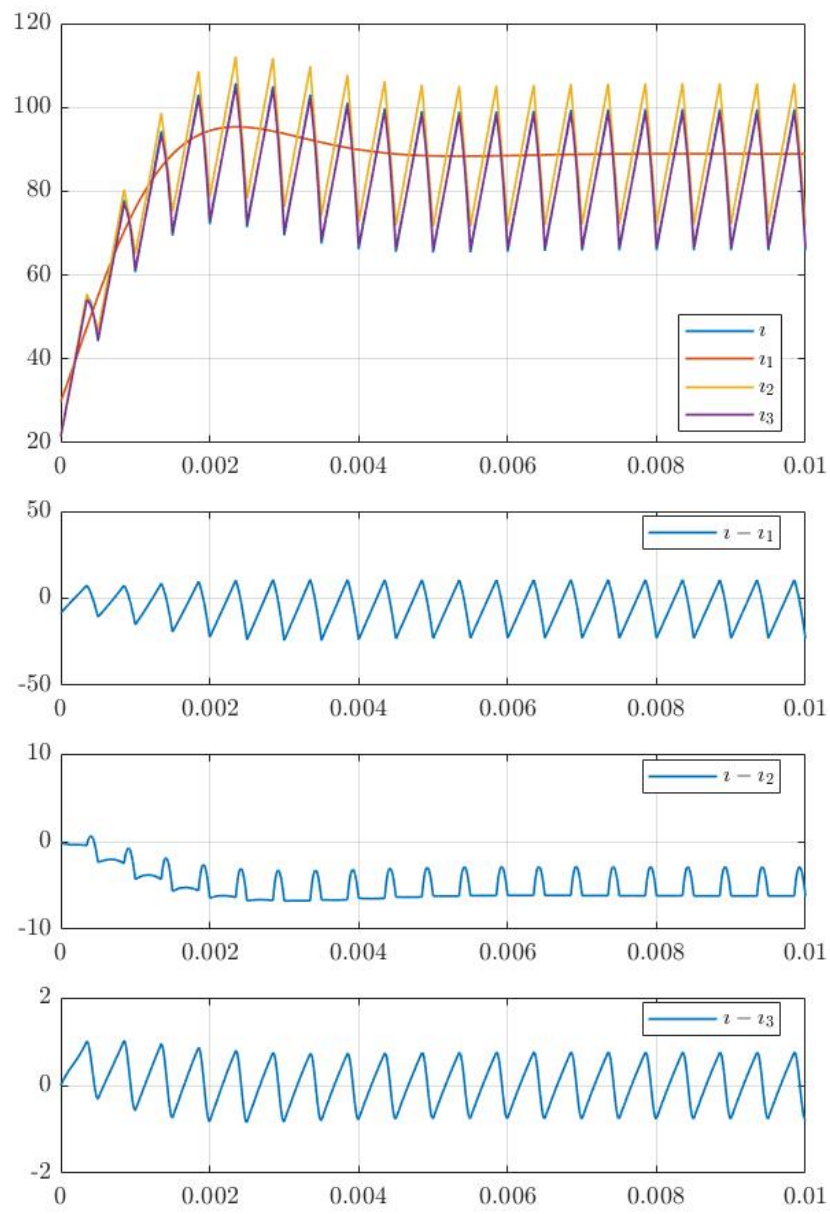

Figure 7. Currents $\imath, \imath_{1}, \imath_{2}, \imath_{3}$ at $2 \mathrm{kHz}$.

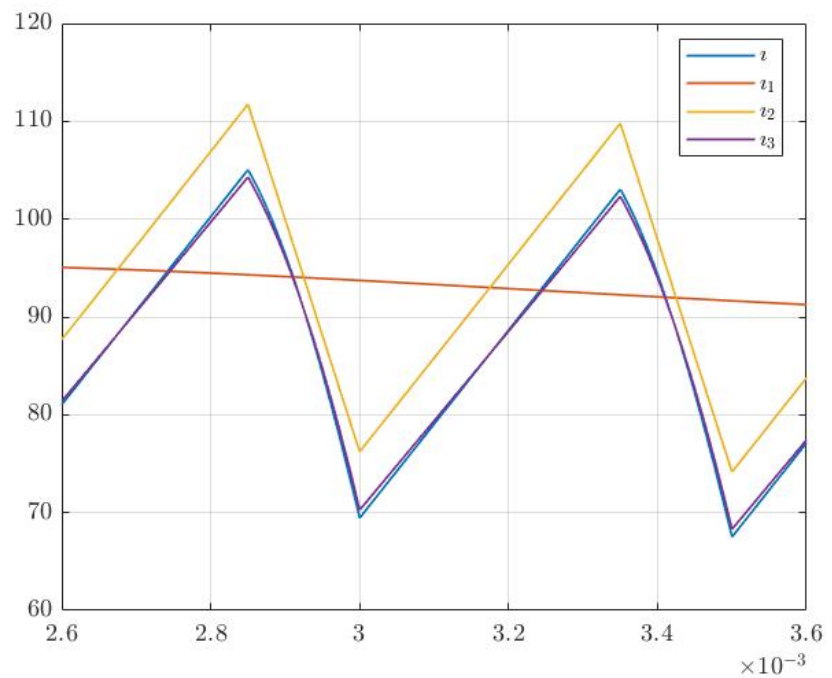

Figure 8. Currents $\imath, \imath_{1}, \imath_{2}, \imath_{3}$ at $2 \mathrm{kHz}$ (zoom). 

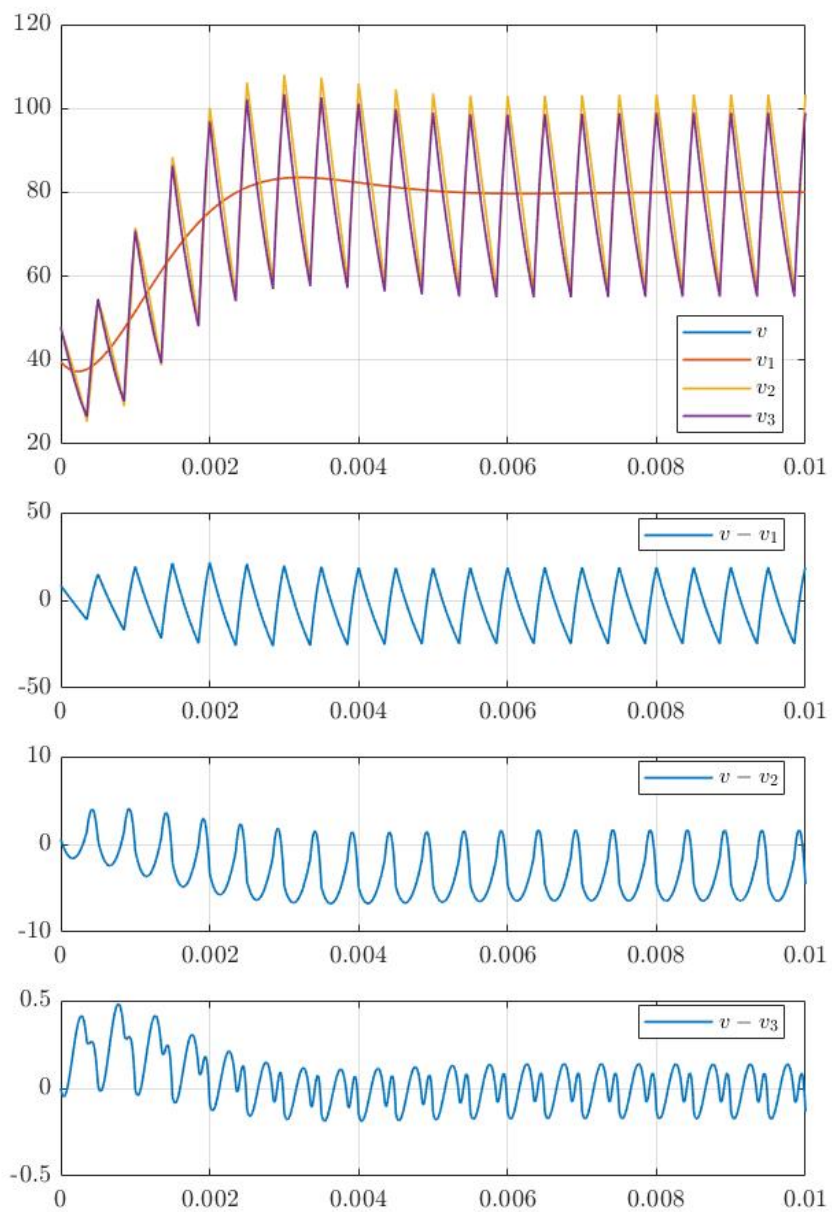

Figure 9. Voltages $v, v_{1}, v_{2}, v_{3}$ at $2 \mathrm{kHz}$.

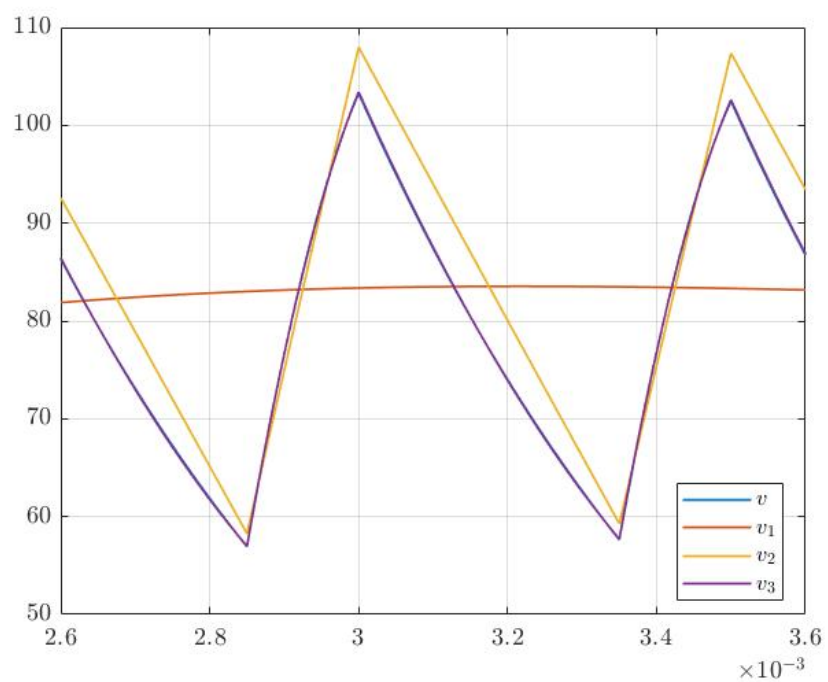

Figure 10. Voltages $v, v_{1}, v_{2}, v_{3}$ at $2 \mathrm{kHz}$ (zoom).
$10^{2}$ times smaller for second-order averaging, and $10^{3}$ times smaller for third-order averaging,

\section{CONCLUSION}

We have presented a revisited derivation of ripple computation in DC-DC converters by higher-order periodic averaging, together with error estimates. The expressions so obtained are very simple for second-order averaging, and still easily tractable for third order averaging. Numerical simulations confirm the relevance of the approach, and the interest of considering third-order averaging when the switching frequency is low.

\section{REFERENCES}

[1] P. Krein, Elements of Power Electronics. Oxford University Press, 1998.

[2] P. Krein, J. Bentsman, R. Bass, and B. Lesieutre, "On the use of averaging for the analysis of power electronic systems," IEEE Transactions on Power Electronics, vol. 5, no. 2, pp. 182-190, 1990.

[3] B. Lehman and R. Bass, "Switching frequency dependent averaged models for pwm dc-dc converters," IEEE Transactions on Power Electronics, vol. 11, no. 1, pp. 89-98, 1996.

[4] — "Extensions of averaging theory for power electronic systems," IEEE Transactions on Power Electronics, vol. 11, no. 4, pp. 542-553, 1996.

[5] V. Caliskan, G. Verghese, and A. Stanković, "Multifrequency averaging of dc/dc converters," IEEE Transactions on Power Electronics, vol. 14, no. 1, pp. 124-133, 1999.

[6] Z. Mihajlovic, B. Lehman, and C. Sun, "Output ripple analysis of switching dc-dc converters," IEEE Transactions on Circuits and Systems I: Regular Papers, vol. 51, no. 8, pp. 1596-1611, 2004.

[7] H. Behjati, L. Niu, A. Davoudi, and P. Chapman, "Alternative time-invariant multi-frequency modeling of pwm dc-dc converters," IEEE Transactions on Circuits and Systems I: Regular Papers, vol. 60 , no. 11, pp. 3069-3079, 2013.

[8] Y. Xu, Y. Chen, C.-C. Liu, and H. Gao, "Piecewise average-value model of pwm converters with applications to large-signal transient simulations," IEEE Transactions on Power Electronics, vol. 31, no. 2, pp. 1304-1321, 2016.

[9] N. N. Bogoliubov and Y. A. Mitropolsky, Asymptotic methods in the theory of non-linear oscillations. Gordon and Breach Science Publishers, 1961, translated from the second revised Russian edition.

[10] J. Sanders, F. Verhulst, and J. Murdock, Averaging methods in nonlinear dynamical systems, 2nd ed. Springer, 2005. 